\title{
Ampliación de programas para adolescentes vulnerables: Experiencias, entendimientos y evidencia
}

Martha Brady

Population Council

Follow this and additional works at: https://knowledgecommons.popcouncil.org/departments_sbsr-pgy

Part of the Demography, Population, and Ecology Commons, Family, Life Course, and Society Commons, Gender and Sexuality Commons, Inequality and Stratification Commons, International Public Health Commons, and the Medicine and Health Commons How does access to this work benefit you? Let us know!

\section{Recommended Citation}

Brady, Martha. 2011. "Ampliación de programas para adolescentes vulnerables: Experiencias, entendimientos y evidencia," Promoción de transiciones a la vida adulta sanas, seguras y productivas Resumen no. 36. New York: Population Council. 


\section{Ampliación de programas para adolescentes vulnerables: Experiencias, entendimientos y evidencia}

\section{Elaborado por Martha Brady}

$\simeq$ pob deseo de la comunidad de desarrollo internacional por aliviar la pobreza y mejorar los resultados de la salud, presenta una oportunidad extraordinaria para transformar la vida de la gente joven, particularmente de las niñas. Ciclos de analfabetismo, escasas posibilidades de trabajo y aislamiento social pueden frenarse, pero requerirán de esfuerzos coordinados para alcanzar a una gran cantidad de niñas adolescentes vulnerables con programas sólidos construidos en base a los valores. El hecho de ampliar los programas piloto efectivos será crítico para lograr estos objetivos. A pesar de que hay un cuerpo de búsqueda en expansión alrededor de intervenciones de ampliación de la salud, se conoce o existen menos documentos acerca de los programas intersectoriales de ampliación para niñas adolescentes. El hecho de traer programas en escala dirigidos a niñas pobres, a menudo despojadas y sordas, posee desafíos únicos. Dado que este es un campo relativamente nuevo en una etapa prematura de implementación, la base de evidencia respecto a que estrategias son más efectivas, aún está emergiendo. El Population Council exploró una variedad de estrategias para alcanzar estos desafíos en entornos diversos y reunió evidencia para notificar el desarrollo y política del programa.

\section{Definiciones y estrategias varias para la ampliación}

La ampliación es un tema de discusión frecuente aunque un estudio relativamente poco riguroso. A pesar de que el término "ampliación" se utiliza ampliamente en la literatura de la salud y el desarrollo, no posee una definición formal. No hay consenso como terminología o marco para estudiar ampliación en el contexto de salud y desarrollo internacional (DeJong 2002; Simmons, Fajians, y Ghiron 2007). "Scaling up" (ampliar) se ha utilizado de forma intercambiable con ìgoing to scaleî (aumentar) y ìat scale" (en escala) y puede abarcar tanto entradas como salidas. Típicamente, estos términos se han utilizado para describir un aumento en el alcance de cobertura y alcance geográfico de una intervención en particular. En el escenario de la salud, "ampliación" generalmente se asocia con resultados específicos, como un acceso incrementado o una demanda por servicios y suministros de salud (Mangham y Hanson 2010). Aún importante, un objetivo para aumentar la cobertura no es suficiente para abordar tantos de los desafíos al

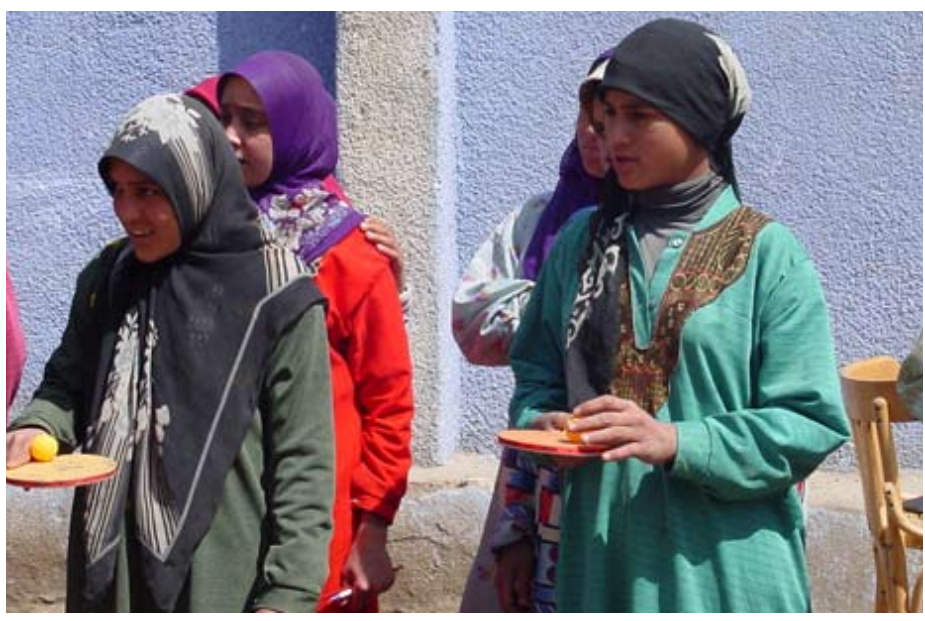

Los programas de ampliación para niñas adolescentes requieren del compromiso de múltiples sectores y de cuidadores diversos.

enfrentarse con niñas adolescentes. Es esencial la atención sobre el contenido y la calidad, así como lo es el proceso de institucionalización.

\section{Ampliación como un proceso de institucionalización}

La ampliación puede además verse como una tarea organizacional, gerencial, política y de política que requiere de un plan y una gestión estratégica (Simmons et al 2007). Es un proceso de institucionalización que abarca múltiples actores, grupos de interés y organizaciones. Dado que la mayoría de los programas de niñas adolescentes son multi-sectoriales, un paso clave es el hecho de identificar la(s) institución(es) apropiada(s) para adoptar la innovación junto al recorrido crítico a ampliar. La ampliación también es un proceso dinámico. En determinadas etapas, el objetivo puede ser aumentar la cobertura, mientras que en otras puede ser incrementar la eficiencia de una iniciativa o programa que ya esté operando a gran escala. Muy a menudo, la ampliación es desigual en términos de ritmo, equidad y alcance. En varios momentos, puede haber tensión entre las dimensiones diferentes de la escala, calidad, cobertura e impacto. (DeJong 2003). Para diseñar una estrategia de ampliación, es importante la apreciación de visiones divergentes de los interesados. 
Observaciones clave alrededor de programas de ampliación de niñas adolescentes

Los investigadores del Population Council están trabajando en una cantidad de entornos para ampliar programas multi-sectoriales de niñas adolescentes. En Bangladesh, Egipto, Etiopía, Guatemala, y Sudáfrica, el Council está trabajando con socios del programa de varios tamaños y capacidad, en diferentes etapas de ampliación y en diversos entornos socio-políticos. A pesar de que el contenido del programa y la población de niñas adolescentes son diversos (ej: rural, niñas casadas en Etiopía, niñas urbanas en Sudáfrica, niñas indígenas en Guatemala, niñas rurales en riesgo de contraer matrimonio prematuro en Bangladesh y niñas fuera de la escuela en el Alto Egipto), las intervenciones comparten principios básicos: crear espacios seguros, comprometer/involucrar a cuidadores de niñas, proporcionarle a las niñas mentores femeninos y ofrecer un enfoque multi-sectorial construido en base a los valores.

\section{Desafíos de los programas de ampliación para niñas vulnerables}

Mientras que la necesidad de ampliar intervenciones efectivas es mucha, los desafíos de realizarlo son considerables. A menudo la capacidad institucional y de gestión para ampliar un programa no está en su lugar; los fondos para ello pocas veces pueden encontrarse en presupuestos del país. Y mientras que se requiera de tiempo para la ampliación, el ritmo también es crítico para mantener el interés de los encargados de formular políticas y de los donantes. El aspecto temporal se destaca particularmente ya que muchos de los resultados que se buscan son a largo plazo. Además, una cantidad de temas esenciales hacen que los programas de ampliación de niñas adolescentes sean excepcionalmente desafiantes. Por ejemplo, a diferencia de otras áreas donde la "demanda" es clara y calculable, en general las niñas no están en posición de articular la demanda de forma que sea oída por los creadores de la política. Muchas niñas están efectivamente fuera de la esfera pública y tienen un acceso limitado a instituciones y recursos de la comunidad. Por consiguiente, el "acceso" a cualquier intervención otorgada requiere de trabajo a través de los cuidadores de las niñas.

Uno de los temas más críticos es identificar un hogar institucional apropiado para la innovación. Debido a que los programas son multi-sectoriales con componentes relacionados a la educación, salud, vida y deportes, no los dejan inmediatamente con solo un "dueño", como un ministro de gobierno. El Council está trabajando con socios a través del espectro, desde pequeñas ONG (Organizaciones no gubernamentales) locales hasta ONG internacionales de gran escala y entidades gubernamentales (locales y nacionales). En el caso de Bangladesh, la ampliación está dentro del contexto de grandes programas de las ONG, mientras que en Etiopía, el programa está extendiendo su alcance a través del gobierno. Tanto en Egipto como en Guatemala, se está adquiriendo un modelo híbrido que capta los sectores públicos y privados. En Sudáfrica, el programa está preparado para ampliarse dentro del contexto del sistema de educación del gobierno.

Transferencia de la innovación del creador al adoptante; roles de la ONG y del gobierno

La ampliación involucra transferir el modelo de un grupo de actores (los creadores) a otro, los "adoptantes." Esto puede llevar varias maneras. Mientras que las ONG a menudo se ven como innovadores sociales que tienen más agilidad y habilidad para realizar un trabajo novedoso, puede presumirse que a la larga el rol para ampliar la innovación sea del sector público. Al mismo tiempo, es ampliamente reconocido que muchos sistemas públicos de gobierno (ej: educación, salud) son débiles. El hecho de ampliar un programa de las ONG puede implicar la expansión organizacional o establecer ramas en otras áreas geográficas o entablar sociedades con otras ONG o cuerpos gubernamentales. En algunos casos las ONG entrenan o financian otras ONG para emprender el trabajo en un campo especializado, mientras que en otros casos han reconocido que la forma más eficaz de alcanzar una escala mayor es capitalizar el alcance y responsabilidad nacional de los gobiernos. Las entidades gubernamentales en general están motivadas por el deseo de alcanzar una proporción significante o incluso todo, de una población dada por razones de equidad, como también de política; el costo y sostenibilidad son preocupaciones críticas para el gobierno.

\section{Mantener la calidad y fidelidad mientras se contienen los costos}

Moverse de un piloto a escala requiere de atención en el contenido, estructura y costo. A menos que se planee cuidadosamente desde el comienzo, los pilotos a menudo son intensivos y costosos, reduciendo la posibilidad de ser adoptados. Por consiguiente, es importante identificar los elementos esenciales para lograr el resultado deseado. El contenido aerodinámico y la entrega son críticos para la contención, viabilidad y sostenibilidad del costo.

\section{Condiciones que aumentan la posibilidad de una ampliación exitosa}

La literatura de difusión-de-innovación (Rogers 1995) proporciona un entendimiento útil acerca de los atributos o condiciones que predicen ampliaciones exitosas. Por ejemplo, hay una posibilidad mayor de éxito si el modelo 0 innovación son:

- Creíbles (basados en pruebas firmes de beneficios)-"prueba del concepto"

- Observables; que beneficiarios previstos (y otros interesados) puedan ver los resultados

- Relevantes (abordar un problema presente o vivido repentinamente)

- Que ofrezcan una ventaja relativa sobre las prácticas o modelos ya existentes

- Fáciles de "instalar" o transferir a entidades de implementación

- Flexibles; que integren nueva evidencia y aprendizaje

- Probables

\section{Abriendo Oportunidades_-"Creating Opportunities"}

El programa Abriendo Oportunidades, implementado por el Population Council y socios locales intersectoriales de las comunidades indígenas de Guatemala, se ha trasladado de un proyecto piloto a un programa cada vez más ampliado con planes para la institucionalización nacional. El programa, el cual crea espacios seguros y oportunidades de liderazgo para las niñas mayas de entre 8 y 18 años, comenzó como un esfuerzo local de ONG en una docena de pueblos y ahora ha alcanzado a más de 3.000 niñas en 40 comunidades indígenas. Abriendo se está expandiendo en un promedio de diez nuevas comunidades por año. Los gobiernos locales, como las ONG y el sector comercial privado, están cada vez más comprometidos y proporcionan las bases necesarias para la sostenibilidad e institucionalización. Además de la expansión del programa en sí mismo, el modelo de Abriendo Oportunidades 


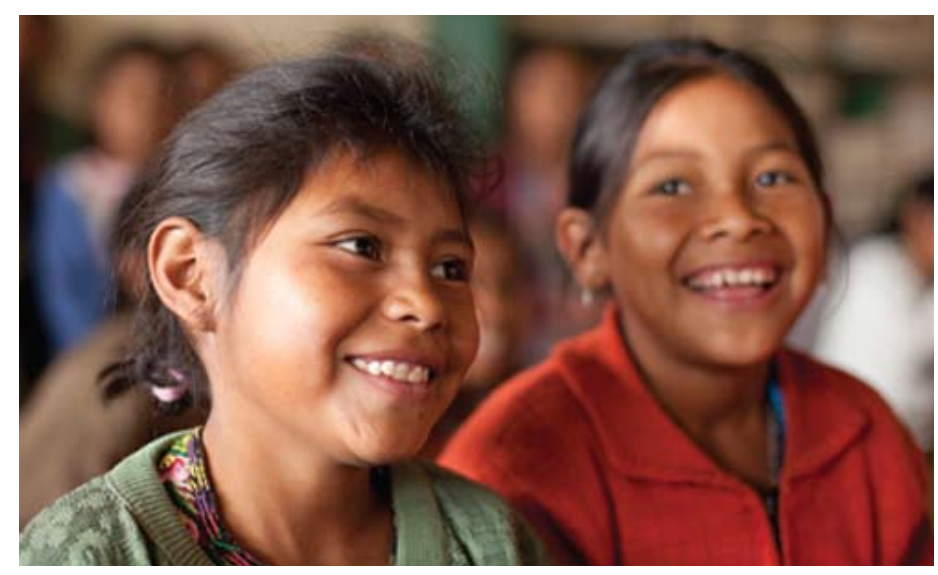

¡Es más que solo números! Los programas de ampliación para niñas adolescentes son un proceso estratégico de institucionalización y la solidificación de los derechos en varias estructuras.

está siendo adoptado e imitado por la ONG pública y los socios del sector privado en Guatemala y está preparado para transformarse en el primer programa nacional para niñas indígenas de la región.

\section{Ishraq-"Sunrise" (Amanecer)}

En Alto Egipto rural, donde las niñas tienen tres veces menos posibilidades de ser inscriptas en la escuela que las niñas de las áreas urbanas, el Population Council y sus socios diseñaron un programa con el objetivo de traer niñas rurales marginadas a espacios seguros de aprendizaje para mejorar su educación, salud y oportunidades sociales. El programa Ishraq enfatiza las habilidades literarias y de vida, la educación de salud e higiene, el compromiso cívico y un primer programa deportivo a nivel gubernamental para niñas. Construyéndose sobre el éxito del piloto en cuatro pueblos con cientos de niñas de entre 13 y 15 años, Ishraq se está ampliando en tres de las gobernaciones más pobres del Alto Egipto.Trabajando a través de ONG locales e internacionales y en colaboración con los socios clave del gobierno que incluyen al National Council of Youth (Consulado Nacional de la Juventud), Ishraq ha alcanzado 30 nuevos pueblos y 1800 niñas que oscilan entre 11 y 15 años.

\section{Berhane Hewan—“Light of Eve" (Luz durante la víspera)}

El Population Council, en colaboración con UNFPA y el Ethiopian Ministry of Youth and Sports (Ministro de Etiopía de Juventud y Deportes), crearon el programa Berhane Hewan (Light of Eve) para asistir a niñas casadas y solteras ligándolas con mentores, redes de soporte social y servicios. Berhane Hewan busca retrasar el matrimonio e incrementar la asistencia a la escuela para niñas vulnerables en Amhara, Etiopía - una región donde la edad media del matrimonio es de 15 años para las niñas (Erulkar y Muthengi 2009). El programa promueve la literatura funcional, aptitudes de vida y la educación de salud reproductiva junto con oportunidades para ahorrar dinero y construir habilidades de vida. Luego de un piloto exitoso que proporcionó evidencia de haber elevado la edad de contraer matrimonio entre las niñas más jóvenes, Berhane Hewan está siendo ampliado a través del gobierno de Etiopía y llega ahora a más de 10.000 niñas a lo largo de la región de Amhara. Una característica clave de este proyecto fue la temprana participación de las unidades del gobierno local (kebeles) y el foco en mantener los costos dentro de una gama que el gobierno pudiera adoptar.
Kishori Abhijan, Bangladesh—“Adolescent Girls' Adventure" (Aventura de niñas adolescentes)

La mayoría de las niñas jóvenes en Bangladesh creen que sus vidas son forzadas debido al matrimonio prematuro. El programa Kishori Abhijan busca disminuir el índice de abandono de la escuela, incrementar la actividad económica independiente de las niñas y aumentar la edad en la que contraigan matrimonio. Como un esfuerzo en conjunto de UNICEF, el United Nations Population Fund (UNFPA) (Fondo de Población de las Naciones Unidas, el Institute of Development Studies (BIDS) (Instituto de Estudios de Desarrollo de Bangladesh), el Rural Advancement Committee (BRAC) (Comité de Avance Rural) de Bangladesh y el Centre for Mass Education in Science (CMES) (Centro de Educación Masiva en Ciencias), el programa Kishori Abhijan combina aptitudes para la vida y entrenamiento de vida. BRAC y CMES, dos ONG grandes y reconocidas, implementaron el programa en 14 distritos rurales, inscribiendo a 15.000 niñas con edades de entre 13 y 22 años. Basado en un piloto exitoso, evaluado por el Population Council y BIDS, el componente de aptitudes para la vida de Kishori Abhijan está siendo ampliado a través de las ONG para inscribir a más de 250.000 niñas en 58 distritos. Con un esfuerzo por construir valores financieros en las niñas, el Council dirigió un programa de educación financiera de 20 horas que ha demostrado resultados prometedores. La participación de la primera generación de los miembros de Kishori Abhijan como ejecutores y mentores del programa en los programas de educación financiera, avala el impacto sostenible y a largo plazo de inversiones tempranas en adolescentes.

\section{Siyakha Nentsha-"Building with Young People" (Construir con gente joven)}

Una intervención respaldada por el Population Council en KwaZulu-Natal, Sudáfrica, incluyó a niños y niñas en un programa aleatorio con capacidades financieras, sociales y de salud basado en la escuela secundaria llamado Siyakha Nentsha, o ỉBuilding with Young Peopleî (Construir con gente joven) en isiZulu. Las niñas fueron el público objetivo debido a su vulnerabilidad por el VIH y embarazos prematuros; los niños fueron incluidos para construir su conocimiento y habilidades y permitir que niños y niñas trabajen en conjunto, interactúen socialmente, se respeten unos a otros como colegas y amigos y así trasladarse más allá de la deshumanización del sexo opuesto. Una encuesta panel que siguió a niños y niñas durante un período de 18 meses, mostró efectos significantes del programa de forma estadística. Como resultado, el Population Council actualmente colabora con el Departamento de Educación de KwaZulu-Natal y con otros socios locales para ampliar el programa en otras escuelas secundarias de la provincia.

\section{Entendimiento durable y lecciones clave}

A la fecha, la mayoría de los éxitos para las niñas han sido pequeños, esfuerzos fragmentados, principalmente fuera de los planes, sistemas y presupuestos del gobierno. El hecho de lograr reacciones de mayor escala requerirá de recursos financieros, técnicos y humanos, junto con la aptitud (las habilidades) y capacidad (la habilidad para gestionar volumen) de hacerlo. Este es particularmente el caso con programas de ampliación de niñas adolescentes, las cuales necesitan de un refuerzo de mensajes y estructuras, un apoyo continuo y liderazgo en todos los niveles. Un resumen de la literatura 
y experiencia de ampliación a través de los programas del Council, ha producido lecciones prácticas que pueden ayudar a guiar los esfuerzos de ampliación para los programas de niñas adolescentes. Por ejemplo:

- Desarrollar una estrategia general para la ampliación que defina los roles, procesos y responsabilidades institucionales y de gestión; es esencial un proceso participativo con interesados relevantes;

- Llevar a cabo una evaluación de la ventaja comparativa al involucrar diferentes tipos de instituciones;

- Usar de la forma más eficaz los sitios piloto e innovadores sociales como modelos; los laboratorios de aprendizaje, las visitas de intercambio y los tours de estudio son mecanismos útiles;

- Mantener y desarrollar un equipo de mentores femeninos para proporcionar el eslabón crítico entre las beneficiarias (niñas) y la gestión del programa; construir capacidad de liderazgo;

- Determinar los elementos del programa que sean centrales para el éxito y los resultados que se buscan;

- Probar la innovación bajo condiciones de "la vida real";

- Construir monitoreo y evaluación en el proceso y

- Seguir aprendiendo, adaptando y reorientando en respuesta a las necesidades y los cambios emergentes del entorno.

Para seguir avanzando: Áreas para investigaciones futuras y apoyo de la política

La inversión en niñas adolescentes tendrá beneficios significantes a largo plazo para la salud, especialmente la salud reproductiva y el desarrollo socio económico. El interés por el rol de las niñas en el desarrollo es fuerte y está en aumento y viene de agencias de la ONU, gobiernos, agencias bilaterales y técnicas y fundaciones. Mientras que el empuje se construye alrededor de programas de ampliación para niñas adolescentes, la investigación para guiar el proceso de ampliación y para medir el impacto de tales esfuerzos es necesaria. Los temas clave para tener en consideración incluyen:

- ¿Cuáles son las expectativas reales para la expansión e institucionalización de una innovación de niñas adolescentes?

- ¿Cuál es el ritmo y alcance apropiados para ampliar la innovación?

- ¿Quiénes gestionarán el proceso de ampliación?

- ¿Cómo se medirá el éxito (ej: sostenibilidad, equidad, alcance)?

- ¿La adaptación del modelo (ej: agregar nuevos elementos, eliminar o modificar) produce los resultados deseados que se observan en el piloto?

- ¿Qué resultados inesperados (positivos o negativos) pueden observarse?

- ¿Cuál es el impacto colectivo?
El Population Council y sus socios seguirán experimentando, refinando y documentando los procesos de ampliación para los programas de niñas adolescentes y buscando oportunidades para compartir entendimiento y evidencia a medida que aparezcan.

\section{Referencias y fuentes}

Cooley, L. y RI Kohl. 2005. "Scale up_From Vision to Large-scale Change: A Management Framework for Practioners," Washington, DC: Management Systems International.

De Jong, J. 2002. A Question of Scale? Expanding NOG Impact in VIHAIDS. Londres: ITDG Publishing.

—. 2003. Making an Impact in VIH and AIDS: NGO Experiences of Scaling up. Londres: ITDG Publishing.

Erulkar, A. y Muthengi, E. 2009. "Evaluation of Berhane Hewan: A program to delay child marriage in rural Ethiopia," International Perspectives on Sexual and Reproductive Health 35(1): 6-14.

ExpandNet. Esta es una red global de profesionales de la salud pública y de científicos que buscan avanzar la práctica y ciencia para ampliar la exitosa innovación del servicio de la salud. Almacenado dentro de WHO, su sitio web incluye cantidad de herramientas y referencias relacionadas a la ampliación: www.expandnet.net

Gilson, L. y Schneider, H. 2010. "Managing scaling up: What are the key issues?" Health Policy and Planning 25: 97-98.

Kohl, R. 2011. "An analytical framework for scaling up pilots in public health" en From One to Many: Scaling up Health Programs in Low Income Countries. University Press Limited, pp. 241-254.

Mangham, L. y Hanson, K. 2010. "Scaling up in international health: What are the issues?" Health Policy and Planning 25: 85-96.

Myers, R. 1992. "Going to scale," in Cooperson, W. (ed.), The Twelve Who Survive. Londres: Routledge, pp.369-396.

Rogers, Everett. 1995. Diffusion of Innovation (4th edition). Nueva York: Free Press.

Shiffman, J. 2007. "Generating political priority for public health causes in developing countries: Implications from a study on maternal mortality." Washington, DC: Center for Global Development.

Simmons, Ruth, Peter Fajians, y Laura Ghiron (eds). 2007. Scaling up Health Service Delivery: From Pilot Innovations to Policies and Programmes. Ginebra: World Health Organization.

Smith, J. y Colvin, C. 2000. "Getting to scale in young adult reproductive health programs." Focus Tool Series No. 3. FOCUS on Young Adults

\section{Donantes}

Population Council

Department for International Development (DFID) (Departamento para el Desarrollo Internacional)

\section{Population Council}

El Population Council cambia la manera en la que el mundo piensa acerca de los problemas de salud y desarrollo. Buscamos entender las causas y las consecuencias de la desigualdad de género y las disparidades en las oportunidades que surgen durante la adolescencia. Brindamos las evidencias para desarrollar mejores programas y políticas prácticas que garanticen una transición exitosa y productiva hacia la edad adulta en los países en vías de desarrollo. www.popcouncil.org

(c) 2011 The Population Council, Inc.

4 - Visite www.popcouncil.org/publications/serialsbriefs/TABriefs.asp para obtener todos los resúmenes de la serie Promoting healthy, safe, and productive transitions to adulthood (Promoción de transiciones a la vida adulta sanas, seguras y productivas). 